\title{
A note on contact metric manifolds
}

\author{
Sharief Deshmukh*1 (D), Amira A. Ishan ${ }^{2}$ (D) \\ ${ }^{1}$ Department of Mathematics, College of science, King Saud University P.O. Box-2455 Riyadh-11451, \\ Saudi Arabia \\ ${ }^{2}$ Department of Mathematics, Taif University, Taif, Kingdom of Saudi Arabia
}

\begin{abstract}
In this paper, first we obtain several necessary and sufficient conditions for a contact metric manifold to be a K-contact manifold and then it is shown that if the Ricci operator of a complete K-contact manifold satisfies a condition like a Codazzi tensor, then it is necessarily a Sasakian manifold.
\end{abstract}

Mathematics Subject Classification (2010). 53C25, 35C20, 35C15

Keywords. contact metric manifolds, K-contact manifolds, Killing vector field, Sasakian manifolds

\section{Introduction}

A contact form $\eta$ on a $(2 n+1)$-dimensional smooth manifold $M$ is a smooth 1-form that satisfies

$$
\eta \wedge(d \eta)^{n} \neq 0
$$

at each point of $M$. The pair $(M, \eta)$ is called a contact manifold. On a contact manifold $(M, \eta)$, there exists a smooth vector field $\xi$ called Reeb vector field which satisfies

$$
\eta(\xi)=1, \xi\rfloor d \eta=0 .
$$

Also, the contact manifold $(M, \eta)$ admits a Riemannian metric $g$ and a skew-symmetric $(1,1)$-tensor field $\varphi$ satisfying

$$
\begin{gathered}
\eta(X)=g(X, \xi), \quad d \eta(X, Y)=2 g(X, \varphi Y), \\
g(\varphi X, \varphi Y)=g(X, Y)-\eta(X) \eta(Y), \quad \varphi(\xi)=0, \\
\eta \circ \varphi=0, \quad \varphi^{2}=-I+\eta \otimes \xi, \quad X, Y \in \mathfrak{X}(M),
\end{gathered}
$$

where $\mathfrak{X}(M)$ is the Lie algebra of smooth vector fields on $M$ (cf. [3]). We call the structure $(\varphi, \xi, \eta, g)$ a contact metric structure and the contact manifold with this structure is denoted by $M(\varphi, \xi, \eta, g)$ and we call it the contact metric manifold (cf. [3]). If in addition, the Reeb vector field $\xi$ of the contact metric manifold is a Killing vector field, then $M(\varphi, \xi, \eta, g)$ is called a $\mathrm{K}$-contact manifold. Note that the Reeb vector field $\xi$ on a K-contact manifold being Killing vector field has its impact on the topology of the Kcontact manifold (cf. [1]). The geometry and topology of a K-contact manifold is studied by several authors (cf. [5-17]). In [12] and [13], the author has used surgery on K-contact

\footnotetext{
*Corresponding Author.

Email addresses: shariefd@ksu.edu.sa (S. Deshmukh), a.ishan@tu.edu.sa (A.A. Ishan)

Received: 10.04.2019; Accepted: 20.03.2020
} 
manifolds to classify 5-dimensional compact simply connected K-contact manifolds and also provided several examples of compact K-contact manifolds. In [8], the author studied the stability of the Reeb vector field of a K-contact manifold with respect to the energy and volume functions treating the Reeb vector field as an embedding of the K-contact manifold into its unit tangent bundle. In [14], it is shown that a necessary and sufficient condition for a contact metric manifold to be a K-contact manifold is that the Reeb vector field is an eigenvector of the Laplacian operator.

A contact metric manifold $M(\varphi, \xi, \eta, g)$ is said to be a Sasakian manifold if it satisfies

$$
(\nabla \varphi)(X, Y)=g(X, Y) \xi-\eta(Y) X, \quad X, Y \in \mathfrak{X}(M),
$$

where $\nabla$ is the Riemannian connection on $M$ and the covariant derivative $(\nabla \varphi)(X, Y)=$ $\nabla_{X}(\varphi Y)-\varphi\left(\nabla_{X} Y\right)$ (cf. [3]). It follows that a Sasakian manifold is a K-contact manifold and the converse is not true (cf. [11]). It is one of the interesting questions in contact geometry to find conditions under which a contact metric manifold is a K-contact manifold and also to find conditions under which a K-contact manifold is a Sasakian manifold. Recently in [5], authors have found one such condition, where they proved that under that condition a compact K-contact Einstein manifold is a Sasakian manifold (cf. Theorem A). Also, in [13] sufficient conditions are found for a contact metric manifold to be a Sasakian manifold.

Let Ric be the Ricci tensor of a Riemannian manifold $(M, g)$. Then the Ricci operator $Q$ is a symmetric $(1,1)$ tensor field defined by (cf. [2])

$$
\operatorname{Ric}(X, Y)=g(Q(X), Y)
$$

and recall that the Ricci operator $Q$ is said to be Codazzi-type operator if it satisfies

$$
(\nabla Q)(X, Y)=(\nabla Q)(Y, X), \quad X, Y \in \mathfrak{X}(M) .
$$

If the Riemannian manifold is an Einstein manifold, then its Ricci operator satisfies the above condition, however the converse is not true, for instance the Ricci operator of the Riemannian product $S^{n}(c) \times S^{m}\left(c_{1}\right)$, with constants $c \neq c_{1}$ satisfies the above condition and is not an Einstein manifold.

A smooth vector field $u$ on a Riemannian manifold $(M, g)$ is said to be a Jacobi-type vector field if it satisfies

$$
\nabla_{X} \nabla_{X} u-\nabla_{\nabla_{X} X} u+R(u, X) X=0, \quad X \in \mathfrak{X}(M),
$$

where $R$ is the curvature tensor field (cf. [7]). A Killing vector field on a Riemannian manifold $(M, g)$ is a Jacobi-type vector field and the converse is not true as there are several examples of Jacobi-type vector fields, which are not Killing (cf. [7]). Jacobi-type vector fields are also used in studying real hypersurfaces of a complex space form (cf. [6], [9]). It is known that a Jacobi-type vector field $u$ on a Riemannian manifold $(M, g)$ satisfies the differential equation

$$
\Delta u+Q(u)=0,
$$

where $\Delta: \mathfrak{X}(M) \rightarrow \mathfrak{X}(M)$ is the Laplacian operator acting on smooth vector fields (cf. [7], Eqn. (2.9)).

One of the interesting questions in contact geometry is to find conditions on a contact metric manifold that makes it a K-contact manifold. There are two ways to answer this question, one using topological restrictions and the other using geometric restrictions. In this paper, we adopt the second option and it has been observed that the Ricci curvature in the direction of the Reeb vector field on a contact metric manifold plays a vital role in tackling this question (cf. Theorem 1). In this paper, we also use the Laplacian operator $\Delta$ acting on smooth vector fields and Jacobi-type vector fields to obtain two necessary and sufficient conditions for a contact metric manifold to be a K-contact manifold (cf. Theorems 2,3) and it has been observed that the characterization in [14] is a particular 
case of Theorem 2. Also, we show that if the Ricci operator of a complete K-contact manifold is Codazzi-type, then it is an Einstein Sasakian manifold and the converse holds (cf. Theorem 4). Finally, we show that the Reeb vector field $\xi$ of a contact metric manifold defines a Ricci soliton on $M$ if and only if it is an Einstein Sasakian manifold. Finally, we consider an ellipsoid in the Euclidean space $R^{4}$ and show that it admits a contact metric structure.

\section{Preliminaries}

Let $M(\varphi, \xi, \eta, g)$ be a $(2 n+1)$-dimensional contact metric manifold. We define a symmetric tensor field $A$ of type $(1,1)$ by setting

$$
\frac{1}{2}\left(£_{\xi} g\right)(X, Y)=g(A X, Y), \quad X, Y \in \mathfrak{X}(M),
$$

where $£_{\xi}$ is the Lie derivative with respect to the vector field $\xi$. Since on a contact metric manifold the contact form $\eta$ satisfies

$$
\frac{1}{2} d \eta(X, Y)=g(X, \varphi Y), \quad X, Y \in \mathfrak{X}(M),
$$

the above two equations together with Koszul's formula (cf. [2]), give

$$
\nabla_{X} \xi=A X-\varphi X, \quad X \in \mathfrak{X}(M) .
$$

Then above equation immediately gives the following expression for the curvature tensor field

$$
R(X, Y) \xi=(\nabla A)(X, Y)-(\nabla A)(Y, X)-(\nabla \varphi)(X, Y)+(\nabla \varphi)(Y, X) .
$$

Define a smooth function $h=\operatorname{Tr} A$, then using skew symmetry of the tensor field $\varphi$, above equation gives

$$
\operatorname{Ric}(Y, \xi)=\sum_{i=1}^{2 n+1}\left[g\left((\nabla A)\left(e_{i}, Y\right), e_{i}\right)-g\left((\nabla \varphi)\left(e_{i}, Y\right), e_{i}\right)\right]-Y(h),
$$

where $\left\{e_{1}, . ., e_{2 n+1}\right\}$ is a local orthonormal frame. Using the symmetry of the operator $A$ and the skew-symmetry of the operator $\varphi$, the above equation gives

$$
Q(\xi)+\nabla h=\sum_{i=1}^{2 n+1}\left[(\nabla A)\left(e_{i}, e_{i}\right)+(\nabla \varphi)\left(e_{i}, e_{i}\right)\right],
$$

where $\nabla h$ is gradient of the smooth function $h$. Now, using

$$
\sum_{i=1}^{2 n+1}(\nabla \varphi)\left(e_{i}, e_{i}\right)=2 n \xi
$$

(cf. equation (3.4)(a) of Lemma 3.1 in [13]), in above equation, we conclude that

$$
Q(\xi)+\nabla h-2 n \xi=\sum_{i=1}^{2 n+1}(\nabla A)\left(e_{i}, e_{i}\right) .
$$

Now, using equation (2.1) and definition of the Laplacian

$$
\left.\Delta \xi=\sum_{i=1}^{2 n+1}\left[\nabla_{e_{i}} \nabla_{e_{i}} \xi-\nabla_{\nabla e_{i} e_{i}} \xi\right)\right]
$$

we get

$$
\Delta \xi=\sum_{i=1}^{2 n+1}\left[(\nabla A)\left(e_{i}, e_{i}\right)-(\nabla \varphi)\left(e_{i}, e_{i}\right)\right]
$$


which in view of equation (3.4)(a) in [13], gives

$$
\Delta \xi+2 n \xi=\sum_{i=1}^{2 n+1}(\nabla A)\left(e_{i}, e_{i}\right)
$$

Then equations (2.4) and (2.5) imply

$$
Q(\xi)+\nabla h-\Delta \xi=4 n \xi
$$

Moreover, in [14], it is shown that on a contact metric manifold $M(\varphi, \xi, \eta, g), Q(\xi)-\Delta \xi=$ $4 n \xi$ holds (cf. Theorem 3.2), here we would caution that our Laplacian operator $\Delta$ is negative of the Laplacian operator used in [14]. Thus, equation (2.6) implies that $\nabla h=0$ and hence, we conclude that on a connected contact metric manifold $M(\varphi, \xi, \eta, g), h=\operatorname{tr} A$ is a constant.

\section{Characterizations of K-contact manifolds}

The geometry of contact metric manifolds, K-contact manifolds, and Sasakian manifolds, though quite classical, yet it is quite important from geometric point of view as well as due to their applications in physics. The geometry of these manifolds is significantly important and in that specially the questions of characterizing K-contact manifolds using a contact metric manifold is very interesting and fascinating. In this section, we prove the first result of this paper, which characterizes K-contact manifolds using a lower bound on the Ricci curvature in the direction of the Reeb vector field.

Theorem 3.1. A $(2 n+1)$-dimensional connected contact metric manifold $M(\varphi, \xi, \eta, g)$ is a K-contact manifold if and only if its Ricci curvature in the direction of the Reeb vector field $\xi$ satisfies

$$
\operatorname{Ric}(\xi, \xi) \geq 2 n
$$

Proof. Suppose $M(\varphi, \xi, \eta, g)$ is a $(2 n+1)$-dimensional contact metric manifold with Ricci curvature $\operatorname{Ric}(\xi, \xi)$ satisfying the given inequality. Since, the symmetric operator $A$ obeys equations (2.1) and that $\operatorname{tr} A=h$, we have

$$
\left\|£_{\xi} g\right\|^{2}=4\|A\|^{2}
$$

and the condition in the statement is

$$
\operatorname{Ric}(\xi, \xi) \geq 2 n \text {. }
$$

Note that the Reeb vector field $\xi$ is a unit vector field and therefore for any smooth vector field $X$ on $M$, we have $X\left(\|\xi\|^{2}\right)=0$, which in view of equation (2.3), gives

$$
g(A X-\varphi X, \xi)=0
$$

and consequently, using $\varphi \xi=0$ in above equation, we conclude

$$
A \xi=0 .
$$

Next, we use equation (2.3), to compute the divergence of the vector field $A \xi$, which leads to

$$
0=\operatorname{div}(A \xi)=\|A\|^{2}+g\left(\xi, \sum_{i=1}^{2 n+1}(\nabla A)\left(e_{i}, e_{i}\right)\right),
$$

where $\left\{e_{1}, . ., e_{n+1}\right\}$ is a local orthonormal frame and we also have used the fact that

$$
\sum_{i=1}^{2 n+1} g\left(A e_{i}, \varphi e_{i}\right)=0 \text {. }
$$


Now, we take the inner product in equation (2.4) with $\xi$ and use equation (3.4) and the fact that $h$ is a constant, to conclude that

$$
\operatorname{Ric}(\xi, \xi)=-\|A\|^{2}+2 n,
$$

that is,

$$
\operatorname{Ric}(\xi, \xi)-2 n=-\|A\|^{2} .
$$

Comparing above equation and the inequality (3.2), we reach to the conclusion $A=$ 0 . Hence, by equation (3.1), we conclude that the Reeb vector field $\xi$ is Killing and consequently, $M(\varphi, \xi, \eta, g)$ is a K-contact manifold.

The converse is trivial, as for a K-contact manifold the Ricci curvature $\operatorname{Ric}(\xi, \xi)=2 n$ (cf. [3], p.67-68).

Recall that the Laplace operator $\Delta: \mathfrak{X}(M) \rightarrow \mathfrak{X}(M)$ acting on smooth vector fields on a Riemannian manifold $(M, g)$ is defined by

$$
\Delta X=\sum_{i=1}^{n}\left(\nabla_{e_{i}} \nabla_{e_{i}} X-\nabla_{\nabla_{e_{i}} e_{i}} X\right),
$$

where $\left\{e_{1}, . . e_{n}\right\}$ is a local orthonormal frame on $M, n=\operatorname{dim} M$. Note that the sign convention used here for the Laplacian operator $\Delta$ is negative of the one used in [14]. Recall that in [14] it is shown that a contact metric manifold $M(\varphi, \xi, \eta, g)$ is a K-contact manifold if and only if the Reeb vector field $\xi$ satisfies $\Delta \xi=-2 n \xi$. Our next result generalizes this result.

Theorem 3.2. $A(2 n+1)$-dimensional connected contact metric manifold $M(\varphi, \xi, \eta, g)$ is a K-contact manifold if and only if the Reeb vector field $\xi$ satisfies

$$
g(\Delta \xi, \xi) \geq-2 n .
$$

Proof. Suppose $M(\varphi, \xi, \eta, g)$ is a $(2 n+1)$-dimensional contact metric manifold with the Reeb vector field satisfying $g(\Delta \xi, \xi) \geq-2 n$. Then taking the inner product in equation (2.5) with $\xi$, leads to

$$
g(\Delta \xi, \xi)+2 n=\sum_{i=1}^{2 n+1} g\left(\xi,(\nabla A)\left(e_{i}, e_{i}\right)\right) .
$$

Using equation (3.4) in above equation, gives

$$
g(\Delta \xi, \xi)+2 n=-\|A\|^{2}
$$

and consequently, we conclude that $A=0$. This proves that $£_{\xi} g=0$, that is, $M(\varphi, \xi, \eta, g)$ is a $\mathrm{K}$-contact manifold.

Conversely, on K-contact manifold $M(\varphi, \xi, \eta, g)$ as $A=0$, the equation (2.5) takes the form

$$
\Delta \xi+2 n \xi=0
$$

which proves $g(\Delta \xi, \xi)=-2 n$ and thus the required condition holds.

As a consequence of above theorem, we have the following, which is essentially proved in [14].

Corollary 3.3. $A(2 n+1)$-dimensional connected contact metric manifold $M(\varphi, \xi, \eta, g)$ is a K-contact manifold if and only if $\Delta \xi=-2 n \xi$ holds. 
Note that the Reeb vector field $\xi$ of a K-contact manifold being Killing is a Jacobi-type of vector field (cf. [7]). Finally, in this section we put a restriction on the Reeb vector field $\xi$ of a contact metric manifold to be a Jacobi-type vector field to find a characterization of a K-contact manifold. Indeed, we prove the following theorem.

Theorem 3.4. A $(2 n+1)$-dimensional connected contact metric manifold $M(\varphi, \xi, \eta, g)$ is a K-contact manifold if and only the Reeb vector field $\xi$ a Jacobi-type vector field.

Proof. Suppose $M(\varphi, \xi, \eta, g)$ is a $(2 n+1)$-dimensional contact metric manifold with the Reeb vector field $\xi$ a Jacobi-type vector field. Since, the Jacobi-type vector field $\xi$ satisfies equation (1.5), we have

$$
Q(\xi)+\Delta \xi=0 .
$$

Now, adding equations (2.4) and (2.5) and noting that $h$ is a constant, we get

$$
Q(\xi)+\Delta \xi=2 \sum_{i=1}^{2 n+1}(\nabla A)\left(e_{i}, e_{i}\right),
$$

which in view of equation (3.5), gives

$$
\sum_{i=1}^{2 n+1}(\nabla A)\left(e_{i}, e_{i}\right)=0 .
$$

Taking the inner product with $\xi$ in above equation and using equation (3.4), gives

$$
\|A\|^{2}=0,
$$

which proves that $M(\varphi, \xi, \eta, g)$ is a K-contact manifold.

The converse is trivial as the Reeb vector field $\xi$ on a K-contact manifold is Killing and is therefore a Jacobi-type vector field.

\section{Characterizations of Einstein Sasakian manifolds}

In this section, we find characterizations of an Einstein Sasakian manifold using a Kcontact manifold as well as contact metric manifold as Ricci soliton. Here in the first characterization the Ricci operator $Q$ plays an important role.

Theorem 4.1. The Ricci operator of a complete K-contact manifold $M(\varphi, \xi, \eta, g)$ satisfies

$$
(\nabla Q)(X, \xi)=(\nabla Q)(\xi, X), \quad X \in \mathfrak{X}(M),
$$

if and only if $M$ is an Einstein Sasakian manifold.

Proof. Let $M(\varphi, \xi, \eta, g)$ be a complete K-contact manifold that satisfies the given condition. Since the Reeb vector field $\xi$ is Killing, its flow $\left\{\psi_{t}\right\}$ consists of local isometries and therefore

$$
d \psi_{t} \circ Q=Q \circ d \psi_{t}
$$

holds. Thus we have

$$
£_{\xi} Q=0,
$$

where $£_{\xi}$ is the Lie derivative with respect to the Reeb vector field. On a K-contact manifold $M(\varphi, \xi, \eta, g)$, as $A=0$, equation (2.3), takes the form

$$
\nabla_{X} \xi=-\varphi X, \quad X \in \mathfrak{X}(M) .
$$

Using equations (4.1) and (4.2), we get

$$
(\nabla Q)(\xi, X)=Q \varphi X-\varphi Q X, \quad X \in \mathfrak{X}(M) .
$$


Now, using the condition on the Ricci operator $Q$ together with equations (4.2) and (4.3), we conclude

$$
\nabla_{X} Q(\xi)=-\varphi Q X, \quad X \in \mathfrak{X}(M) .
$$

Recall that on a K-contact manifold $M(\varphi, \xi, \eta, g)$, we have $Q(\xi)=2 n \xi$, where $\operatorname{dim} M=$ $2 n+1$ and consequently, equations (4.2) and (4.4) give

$$
\varphi Q(X)=2 n \varphi X, \quad X \in \mathfrak{X}(M) .
$$

On operating $\varphi$ on the above equation and using $Q(\xi)=2 n \xi$, we get $Q(X)=2 n X$, $X \in \mathfrak{X}(M)$, that is, the K-contact manifold is an Einstein manifold. Observe that the Ricci curvature is strictly positive and therefore by Myers' theorem the K-contact manifold is compact. This proves the K-contact manifold is a compact Einstein manifold and therefore an Einstein Sasakian manifold by Theorem A in [4].

The converse is trivial.

Suppose the Reeb vector field $\xi$ of a contact metric manifold $M(\varphi, \xi, \eta, g)$ is the potential vector field that defines a Ricci soliton [5]. Then the Ricci tensor satisfies

$$
\text { Ric }+\frac{1}{2} £_{\xi} g=\lambda g,
$$

where $\lambda$ is a constant. We get the following characterization of Einstein Sasakaian manifolds.

Theorem 4.2. $A(2 n+1)$-dimensional complete and connected contact metric manifold $M(\varphi, \xi, \eta, g)$ is an Einstein Sasakian manifold if and only if the Reeb vector field $\xi$ defines a Ricci soliton on $M$.

Proof. Let $M(\varphi, \xi, \eta, g)$ be a complete and connected contact metric manifold and $\xi$ be the potential field of the Ricci soliton structure on $M$. Then by equations (2.1) and (4.5), we get

$$
A X=\lambda X-Q X, \quad X \in \mathfrak{X}(M),
$$

which gives $\lambda(2 n+1)-S=h$, where $h=\operatorname{tr} A$ is a constant. Using equation (4.6), we have

$$
\sum_{i=1}^{2 n+1}(\nabla A)\left(e_{i}, e_{i}\right)=-\sum_{i=1}^{2 n+1}(\nabla Q)\left(e_{i}, e_{i}\right)=-\frac{1}{2} \nabla S=0,
$$

which in view of equation (2.5), gives $\Delta \xi=-2 n \xi$ and this proves that $M(\varphi, \xi, \eta, g)$ is a K-contact manifold, that is the Reeb vector field $\xi$ is Killing and that $\operatorname{Ric}(\xi, \xi)=2 n$. Also, as $\xi$ is Killing, equation (4.5) gives Ric $=\lambda g$, that is, the K-contact manifold is an Einstein manifold with $\lambda=2 n$. Moreover, as the Ricci curvature is strictly positive by Myers' theorem the K-contact manifold is compact. This proves the K-contact manifold is a compact Einstein manifold and therefore an Einstein Sasakian manifold by Theorem $\mathrm{A}$ in [4].

The converse is trivial as the Reeb vector field of an Einstein Sasakian manifold satisfies (4.5) with $\lambda=2 n$.

\section{An example}

In this section, we give a new example of a contact metric manifold. Indeed, we consider an ellipsoid $M$ in the Euclidean space $\boldsymbol{R}^{4}$ and show the existence of smooth 1-form $\eta$ on $M$ that defines a contact structure that does not give a contact metric structure with respect to the induced metric $g$ on the hypersurface $M$. Then we find other metric $\bar{g}$ and find its expression, which gives a contact metric structure $(\varphi, \xi, \eta, \bar{g})$ on $M$. 
Consider a smooth function $h: \boldsymbol{R}^{4} \rightarrow \boldsymbol{R}, h(x, y, z, w)=x^{2}+y^{2}+4 z^{2}+4 z^{2}-1$. Then $M=h^{-1}(0)$ is a compact hypersurface of the Euclidean space $\left(\boldsymbol{R}^{4},\langle\rangle,\right)$ with induced metric $g$ and unit normal

$$
N=\frac{1}{f}(x, y, 4 z, 4 w), \quad f=\sqrt{x^{2}+y^{2}+16 z^{2}+16 w^{2}} .
$$

Note that $f \neq 0$ on $M$ as $f=\sqrt{1+12 z^{2}+w^{2}}$. Now, consider the unit vector field $\xi=(-y, x,-2 w, 2 z)$ on the Riemannian manifold $(M, g)$ with dual smooth 1-form

$$
\eta=-y d s+x d y-2 w d z+2 z d w .
$$

Then, it follows that $\xi(f)=0$ and

$$
d \eta=2(d x \wedge d y+2 d z \wedge d w)
$$

$\eta \wedge d n=4(-y d x \wedge d y \wedge d w+x d y \wedge d z \wedge d w-w d x \wedge d y \wedge d z+z d x \wedge d y \wedge d w)$.

If for a point $p \in M,(\eta \wedge d n)(p)=0$, we get $x(p)=y(p)=z(p)=w(p)=0$, which gives $h(p)=-1$, a contradiction. Hence, $\eta \wedge d n \neq 0$, that is, $\eta$ is a contact form on $(M, g)$. Moreover, for $X \in \mathfrak{X}(M)$ we have

$$
\begin{aligned}
d \eta(\xi, X) & =2(d x \wedge d y+2 d z \wedge d w)(\xi, X) \\
& =\xi(x) X(y)-\xi(y) X(x)+2 \xi(z) X(w)-2 \xi(w) X(z) \\
& =-\frac{1}{2} X\left(x^{2}+y^{2}+4 z^{2}+4 w^{2}\right)=0 .
\end{aligned}
$$

Define a skew symmetric $(1,1)$ tensor field $\varphi$ required for a contact metric structure by $2 g(X, \varphi Y)=d \eta(X, Y)$ on $M$. Then the last equation, gives $\varphi(\xi)=0$. We claim that $(M, \varphi, \xi, \eta, g)$ is not a contact metric manifold. To establish the claim, first we show that $\xi$ is a Killing vector field on $(M, g)$ and thus if $(M, \varphi, \xi, \eta, g)$ is a contact metric manifold, it will be a K-contact manifold and as a 3-dimensional K-contact manifold is a Sasakian manifold and that will force the sectional curvatures of plane sections containing the Reeb vector field $\xi$ to be 1, which does not happen for the hypersurface with the induced metric.

Let $D$ be the Euclidean connection on the Euclidean space $\left(\boldsymbol{R}^{4},\langle\rangle,\right)$ and $\nabla$ be the Riemannian connection on the hypersurface $(M, g)$ and $A$ be shape operator of the hypersurface. Then we get

$$
A \xi=-D_{\xi} N=\frac{1}{f}(y,-x, 8 w,-8 z),
$$

where we used $\xi(f)=0$. Consequently, we compute

$$
\begin{aligned}
\nabla_{X} \xi & =D_{X} \xi-g(A \xi, X) N \\
& =-\left(X(y)+\frac{x \delta}{f^{2}},-X(x)+\frac{y \delta}{f^{2}}, 2 X(w)+\frac{4 z \delta}{f^{2}},-2 X(z)+\frac{4 w \delta}{f^{2}}\right),
\end{aligned}
$$

where $\delta=y X(x)-x X(y)+8 w X(z)-8 z X(w)$. We get

$$
\begin{aligned}
g\left(\nabla_{X} \xi, Y\right)= & -(X(y) Y(x)-X(x) Y(y)+2 X(w) Y(z)-2 X(z) Y(w)) \\
& -\frac{\delta}{f^{2}}(x Y(x)+y Y(y)+4 z Y(z)+4 w Y(w)) .
\end{aligned}
$$

Using $x Y(x)+y Y(y)+4 z Y(z)+4 w Y(w)=\frac{1}{2} Y\left(x^{2}+y^{2}+4 z^{2}+4 z^{2}\right)=0$, in above equation, we get

$$
g\left(\nabla_{X} \xi, Y\right)=-(X(y) Y(x)-X(x) Y(y)+2 X(w) Y(z)-2 X(z) Y(w))
$$

and consequently, that

$$
\left(£_{\xi} g\right)(X, Y)=0, \quad X, Y \in \mathfrak{X}(M) .
$$


Hence, $\xi$ is a Killing vector field, that makes $(M, \varphi, \xi, \eta, g)$ a 3 -dimensional K-contact manifold and consequently is a Sasakian manifold. Now, we compute sectional curvature of a plane section containing $\xi$. Take a unit vector field $\bar{\xi}$ on $M$ given by

$$
\bar{\xi}=\frac{1}{f}(-y, x,-4 w, 4 z),
$$

then we get $g(\xi, \bar{\xi})=\frac{1}{f}\left(x^{2}+y^{2}+8 z^{2}+18 w^{2}\right)$ and $\bar{\xi}(f)=0$ and

$$
A \bar{\xi}=\frac{1}{f^{2}}(y,-x, 16 w,-16 z) \text {. }
$$

Thus, we have

$$
\begin{aligned}
& g(A \xi, \xi)=-f, \quad g(A \bar{\xi}, \bar{\xi})=-\frac{1}{f^{3}}\left(x^{2}+y^{2}+64 z^{2}+64 w^{2}\right) \\
& g(A \xi, \bar{\xi})=-\frac{1}{f^{2}}\left(x^{2}+y^{2}+32 z^{2}+32 w^{2}\right)
\end{aligned}
$$

and consequently, the sectional curvature $K(\xi, \bar{\xi})$ of the hypersurface $(M, g)$ is given by

$$
K(\xi, \bar{\xi})=\frac{1}{|\xi \wedge \bar{\xi}|^{2}} R(\xi, \bar{\xi} ; \bar{\xi}, \xi)=\frac{1}{|\xi \wedge \bar{\xi}|^{2}}\left(g(A \xi, \xi) g(A \bar{\xi}, \bar{\xi})-g(A \xi, \bar{\xi})^{2}\right)
$$

Using above equations, after some computations, we conclude

$$
K(\xi, \bar{\xi})=\frac{16}{f^{4}|\xi \wedge \bar{\xi}|^{2}}\left(x^{2}+y^{2}\right)\left(z^{2}+w^{2}\right),
$$

where

$$
|\xi \wedge \bar{\xi}|^{2}=1-\frac{1}{f^{2}}\left(x^{2}+y^{2}+8 z^{2}+8 w^{2}\right)^{2} .
$$

Now taking a point $p=\left(\frac{1}{\sqrt{4}}, \frac{1}{\sqrt{4}}, \frac{1}{2 \sqrt{4}}, \frac{1}{2 \sqrt{4}}\right) \in M$, and above equation, we find that the sectional curvature $K(\xi, \bar{\xi})(p)=\frac{16}{5} \neq 1$, that is a contradiction to $(M, \varphi, \xi, \eta, g)$ being a Sasakian manifold. Hence, $(M, \varphi, \xi, \eta, g)$ is not a contact metric manifold. Finally, we proceed to find the expression for the metric $\bar{g}$, that makes $(M, \varphi, \xi, \eta, \bar{g})$ a contact metric manifold. Since, $(M, \varphi, \xi, \eta, \bar{g})$ is a contact metric manifold, using equation (5.3), we have

$$
2 \bar{g}(X, \varphi Y)=d \eta(X, Y)=2(d x \wedge d y+2 d z \wedge d w)(X, Y), \quad X, Y \in \mathfrak{X}(M) .
$$

Note that using expression for $\eta$, we get $\eta(X)=-y X(x)+x X(y)-2 w X(z)+2 z X(w)$. Thus, replacing $Y$ by $\varphi Y$ and using equation (1.1) in above equation, we get

$$
\begin{aligned}
\bar{g}(X, Y)= & X(x)\left(-y \eta(Y)-\frac{1}{2}(\varphi Y)(y)\right)+X(y)\left(x \eta(Y)+\frac{1}{2}(\varphi Y)(x)\right) \\
& +X(z)(-2 w \eta(Y)-\varphi(Y)(w))+X(w)(2 z \eta(Y)+\varphi(Y)(z)) .
\end{aligned}
$$

Acknowledgment. The authors extend their appreciations to the Deanship of Scientific Research, King Saud University for funding this work through research group no (RG-1441P182). 


\section{References}

[1] V. Berestovskii and Y. Nikonorov, Killing vector fields of constant length on Riemannian manifolds, Siberian Math. J. 49 (3), 395-407, 2008.

[2] A.L. Besse, Einstein Manifolds, Springer Verlag, 1987.

[3] D.E. Blair, Contact Manifolds in Riemannian Geometry, Lecture Notes in Math. 509, Springer Verlag, 1976.

[4] C. Boyer and K. Galicki, Einstein manifolds and contact geometry, Proc. Amer. Math. Soc. 129 (8), 2419-2430, 2001.

[5] B. Chow, P. Lu and L. Ni, Hamilton's Ricci Flow, Graduate studies in Mathematics, 77, AMS Scientific Press, 2010.

[6] S. Deshmukh, Real hypersurfaces of a complex space form, Proc. Math. Sci. 121 (2), 171-179, 2011.

[7] S. Deshmukh, Jacobi-type vector fields on Ricci solitons, Bull. Math. Soc. Sci. Math. Roumanie 55 (103), No. 1, 41-50, 2012.

[8] A. Hurtado, Stability numbers in K-contact manifolds, Diff. Geom. Appl. 26 (3), 227-242, 2008.

[9] C.J.G. Manchado and J.D. Perez, On the structure vector field of a real hypersurface in complex two-plane Grassmannians, Cent. Eur. J. Math. 10 (2), 451-455, 2012.

[10] A. Mastromartino and Y. Villarroel, The annihilator of a K-contact manifold, Math. Rep. (Bucur.) 6 (56), 431-443, 2004.

[11] B.C Montano, A.D. Nikola, J.C. Marrero, and I. Yudin, Examples of compact Kcontact manifolds with no Sasakian metric, Int. J. Geom. Methods Mod. Phys. 11 (9), 1460023, 10 pp., 2014.

[12] M. Okumura, Certain almost contact hypersurfaces in Kaehler manifolds of constant holomorphic sectional curvature, Tohoku Math. J. (2), 16, 270-284, 1964.

[13] Z. Olszak, On contact metric manifolds, Tohoku Math. J. (2), 31, 247-253, 1979.

[14] D. Perrone, Contact metric manifolds whose characteristic vector field is harmonic vector field, Differential Geom. Appl. 20, 367-378, 2004.

[15] T. Yamazaki, On a surgery of K-contact manifolds, Kodai Math. J. 24 (2), 214-225, 2001.

[16] T. Yamazaki, A construction of K-contact manifolds by a fiber join, Tohoku Math. J. (2), 51 (4), 433-446, 1999.

[17] A. Yildiz and E. Ata, On a type of K-contact manifolds, Hacet. J. Math. Stat. 41 (4), 567-571, 2012. 